\title{
Large scale diffuse light in the Coma cluster: A multi-scale approach
}

\author{
C. Adami ${ }^{1}$, E. Slezak ${ }^{2}$, F. Durret ${ }^{3}$, C. J. Conselice ${ }^{4}$, J. C. Cuillandre ${ }^{5}$, J. S. Gallagher ${ }^{6}$, A. Mazure ${ }^{1}$, \\ R. Pelló ${ }^{7}$, J. P. Picat ${ }^{7}$, and M. P. Ulmer ${ }^{8}$
}

${ }^{1}$ LAM, Traverse du Siphon, 13012 Marseille, France

e-mail: christophe.adami@oamp.fr

2 Observatoire de la Côte d'Azur, BP 4229, 06304 Nice Cedex 4, France

3 Institut d'Astrophysique de Paris, CNRS, Université Pierre et Marie Curie, 98bis Bd Arago, 75014 Paris, France

4 Department of Astronomy, Caltech, MS 105-24, Pasadena CA 91125, USA

5 Canada-France-Hawaii Telescope Corporation, 65-1238 Mamalahoa Highway, Kamuela, HI 96743

${ }^{6}$ University of Wisconsin, Department of Astronomy, 475 N. Charter St., Madison, WI 53706, USA

7 Observatoire Midi-Pyrénées, 14 Av. Edouard Belin, 31400 Toulouse, France

8 NWU, Dearborn Observatory, 2131 Sheridan, 60208-2900 Evanston, USA

Received 19 May 2004 / Accepted 23 July 2004

\begin{abstract}
We have obtained wide field images of the Coma cluster in the $B, V, R$ and $I$ bands with the CFH12K camera at CFHT. To search for large scale diffuse emission, we have applied to these images an iterative multiscale wavelet analysis and reconstruction technique which made it possible to model all the sources (stars and galaxies) and subtract them from the original images. We found various concentrations of diffuse emission present in the central zone around the central galaxies NGC 4874 and NGC 4889. We characterize the positions, sizes and colors of these concentrations. Some sources do not seem to have strong star formation, while one probably exhibits spiral-like colors. One possible origin for the star forming diffuse emission sources is that in the region of the two main galaxies NGC 4874 and NGC 4889 spiral galaxies have recently been disrupted and star formation is still active in the dispersed material. We also use the characteristics of the sources of diffuse emission to trace the cluster dynamics. A scenario in which the group around NGC 4874 is moving north is consistent with our data.
\end{abstract}

Key words. galaxies: clusters: individual: Coma cluster: Abell 1656 - galaxies: clusters: general

\section{Introduction}

Diffuse emission in clusters of galaxies may put strong constraints on cluster formation processes, as well as on the mechanisms that might account for the enrichment of the intergalactic medium such as galaxy harassment or stripping.

The existence of intergalactic matter visible at optical wavelengths in the Coma cluster was already reported by Zwicky (1951), and several searches based on photographic plate material were undertaken in the 1970's on Abell 2670 (Oemler 1973) and Coma (Thuan \& Kormendy 1977). Interestingly, Thuan \& Kormendy (1977) already noted that the diffuse light appeared to be bluer than the galaxies, a fact consistent with the idea that it is made of stars tidally stripped from galaxies, which are usually bluer at large radii. Later on, CCDs made it possible to better model and subtract galaxies (including the giant central galaxy) from images to obtain the diffuse light distribution after applying various treatments (Gudehus 1989; Uson et al. 1991; Vílchez-Gómez et al. 1994; Gonzalez et al. 2000).
Besides, there has also been an intensive search for various types of stars which could belong to an intergalactic population, such as planetary nebulae in Virgo (Arnaboldi et al. 1996, 2003; Feldmeier et al. 1998) and in Fornax (Theuns \& Warren 1997), red giants in Virgo (Durrell et al. 2002) or the progenitors of two supernovae in Abell 403 and Abell 2122/4 (Gal-Yam et al. 2003). A global result is that these stars could contribute up to 5-20\% of the cluster total luminosity, thus making this component an important one when analyzing the optical properties of clusters.

The idea that diffuse light in clusters is probably due to tidal disruptions of galaxies has recently been confirmed numerically by Calcáneo-Roldán et al. (2000). These authors have applied numerical simulations to show that the arc of diffuse light detected in the Centaurus cluster is probably the debris of a tidally disrupted spiral galaxy. With the purpose of observing more tidal debris in clusters, deep imaging surveys have recently been initiated which show evidence for plumes and arclike structures (Feldmeier et al. 2002, 2003; Mihos 2003; Gregg \& West 1998). 
We have undertaken an extensive analysis of the Coma cluster, based on deep multiband imaging $(B, V, R$ and $I$ filters) of a region of $42 \times 50 \mathrm{arcmin}^{2}$ which will be presented in subsequent papers. To search for diffuse light features we used a wavelet analysis and reconstruction of the image to recover and subtract all the objects (stars and galaxies) from the raw image. We will compare the diffuse features revealed by our treatment to those found by Gregg \& West (1998). Note that Trentham \& Mobasher (1998) also reported the presence of a number of low surface brightness features in Coma.

The data will be briefly presented in Sect. 2 and our method in Sect. 3. Results will be presented in Sects. 4 and 5. A possible scenario to explain our results will be presented and discussed in Sect. 6 and conclusions are summarized in Sect. 7.

\section{The data}

The full set of data at our disposal is described in a companion paper (Savine et al. in preparation). We list however the main points.

Images were obtained at the $3.6 \mathrm{~m} \mathrm{CFH}$ telescope in the spring of 1999 and of 2000 with the CFH12K CCD camera of $12365 \times 8143$ pixels $^{2}$, with a scale of 0.206 arcsec/pixel in the CFH12K $B, V, R$ and $I$ filters (close to Johnson filters but not identical), for an area of about $42 \times 50 \mathrm{arcmin}^{2}$ centered on the dominant galaxies of the Coma cluster, NGC 4874 and NGC 4889. This area was covered by two CFH12K fields which overlap by $\sim 7^{\prime}$ in the North/South direction. Total exposure times were $7200 \mathrm{~s}$ in 5 exposures for the $B$ band, $5040 \mathrm{~s}$ in 7 exposures for the $V$ band and the North field, $2400 \mathrm{~s}$ in 5 exposures for the $V$ band and the South field, $3300 \mathrm{~s}$ in 6 exposures for the $\mathrm{R}$ band and the South field and $3600 \mathrm{~s}$ in 5 exposures for the $R$ band and the North field. Superflat fields were used (combinations of several empty exposures delivered as a final product by the CFHT pipeline). The $B, V, R$ and $I$ data are complete down to $R \sim 24$. Final image qualities range from 0.85 to 1 arcsec.

In this paper we choose to use only $B, V$ and $R$ band data for the whole field of view. We discarded the $I$ band data which present some residual artefacts from the sky subtraction. We analyzed the $R$ band data, which are the deepest, in the whole field of view and, the $B$ and $V$ band data only in the regions of interest, where diffuse emission is detected in the $R$ band.

\section{Data analysis}

\subsection{Method}

Our aim is to detect large scale faint diffuse light emission sources in unknown locations of an astronomical image in the presence of structures (stars and galaxies) of different scales. The usual way is to remove these luminous objects. An attractive approach is to perform a multiscale analysis of the image, assuming that the spatial extension of the diffuse sources is in most cases larger than the typical star or galaxy scales and just keep the large scale components. No prior identification or modeling of the structures to be subtracted are required as was done for instance by Vílchez-Gómez et al. (1994). The efficiency of the multiscale analysis approach depends of course on the initial assumption for the separation criterion between different structures, but in any case the noise reduction due to the method should make easier the retrieval of diffuse light structures with scales comparable to those of stars and galaxies.

However, large scale saturated star haloes remain a problem. Such features are so extended that removing completely their contributions would require using very large scales in the treatment described below, and this would also remove large scale diffuse light sources. When possible, we therefore excluded the regions occupied by these very bright stars (two out of three).

To obtain a denoised image of the objects smaller than a maximum size we applied the method described in Bijaoui \& Rué (1995) and Durret et al. (2002) with some modifications that improved the quality of the final result. We will only recall the salient points of the treatment. The method involves a thresholding of the data in the wavelet space in order to remove the noise locally without smoothing the astrophysical signal. This is explained by the fact that, since wavelets are functions with a mean value equal to zero, a continuous object in real space appears at any scale in the wavelet space as a region of positive wavelet coefficients surrounded by a border of negative coefficients. It appears that the characteristics of the object, which can be computed from the subset of coefficients that are statistically significant with respect to the noise rms scatter, are better estimated when these negative values are also considered, especially for bright objects.

Note that wavelet coefficients are considered to be significant at a given scale when their absolute magnitude exceeds 3 times the rms fluctuation expected for the wavelet coefficients of white noise at this same scale. Larger thresholds are introduced for the first (smallest) and second scales (4.0 and 3.5 times the rms fluctuations respectively) in order to remove most of the spurious unavoidable false alarms at these scales. Hence, we first decided to select not only positive but also negative significant wavelet coefficients when thresholding the wavelet transform of the image.

Second, negative coefficients for one object locally decrease the positive coefficients linked to any object close enough to this first object. When detecting real structures in a noisy image by means of their corresponding domains in the wavelet space, faint objects close to a bright object can therefore easily be missed. This can be a serious problem because we have in our catalog faint objects distributed in an area with very bright objects in the center (as expected for a cluster of galaxies). Consequently, in order to carry out a more complete survey of objects within a given size range, we decided to perform the analysis twice. From the first subset of significant wavelet coefficients, a positive image in real space is restored using an iterative conjugate gradient algorithm with a regularization constraint (Appendix A in Rué \& Bijaoui 1997). This image is subtracted from the original one and the wavelet transform of the residual is thresholded with similar thresholds as in the first iteration. Applying the restoration algorithm to the second subset of coefficients enables one to get another image including the previously hidden significant features (see Fig. 1d). 
(a)

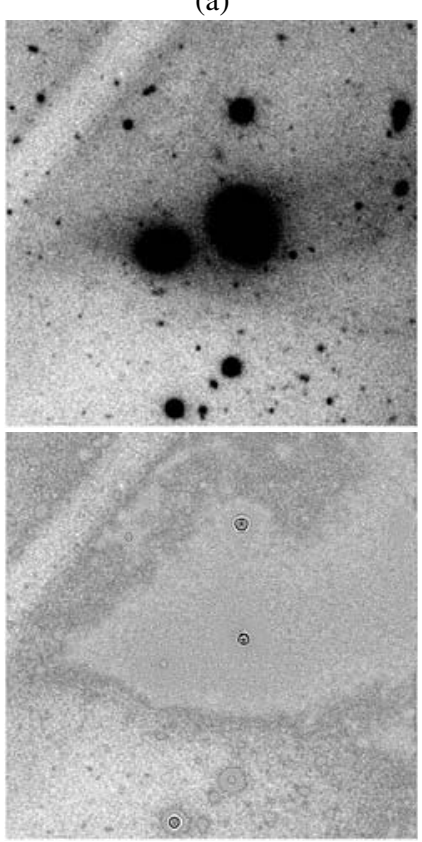

(c) (b)

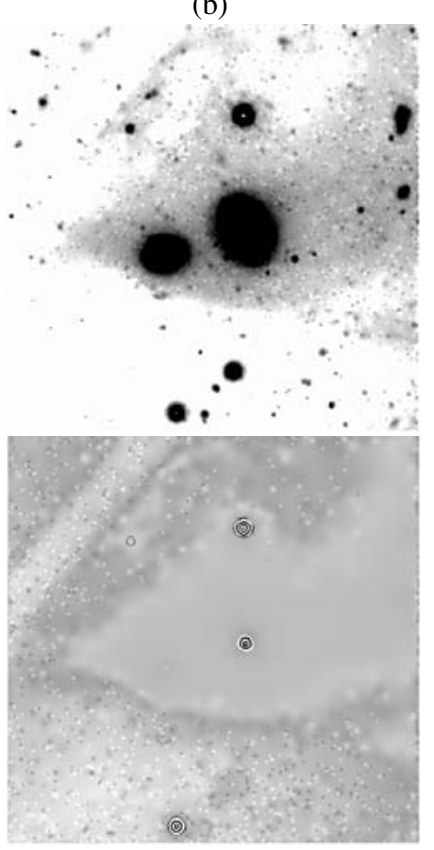

(d)

Fig. 1. A two step methodology is required to detect and restore faint features close to bright ones with wavelets. All images are $1.75^{\prime} \times$ $1.75^{\prime}$ in the $R$ band. a) Original image with objects of different shapes and surface brightnesses partly superimposed on the halo of a very bright star in the neighborhood; b) image restored from a thresholded wavelet transform selecting coefficients in the scale range $2^{0}-2^{9}$ pixels in radius with an absolute value larger than 3 times the rms scatter expected for pure noise - some faint features have been missed; c) image obtained by subtracting the denoised image from the original one, showing the faint features at various scales which have been missed at the first step as well as some annular restoration artefacts due to local saturation effects; d) denoised image of the residual image computed from the subset of its wavelet coefficients in the scale range $2^{0}-2^{7}$ pixels found significant with respect to the same thresholds as in the first step.

In this way, adding together the two restored images with a positivity constraint for the result (cf. the subtraction step) enables one to obtain a more adequate map of the small scale structures within the image. This last map can then be subtracted from the original image, leading to a new residual image where large scale emission components can be quite easily searched for. Hence, the final products of this process are an image of what we call the objects, i.e. the signal with a characteristic scale smaller than the maximal scale used, and a residual image exhibiting mostly features with a characteristic scale larger than this maximum value (see Fig. 2).

\subsection{Application}

The images to be examined contain the two dominant galaxies of the Coma cluster of galaxies as well as three very bright stars in the northern field. To handle these large objects we decided to perform the first wavelet transform of our analysis using ten scales (from a minimum scale $a=2^{0}=1$ pixel to a maximum scale $a=2^{9}=512$ pixels). Features with a characteristic size

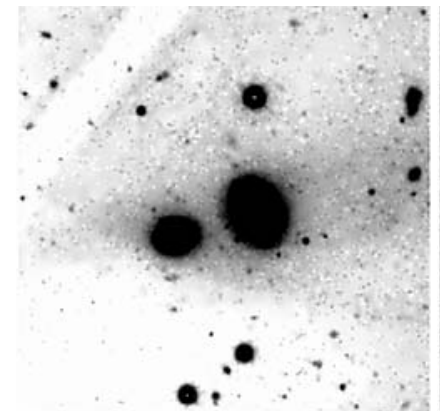

(a)

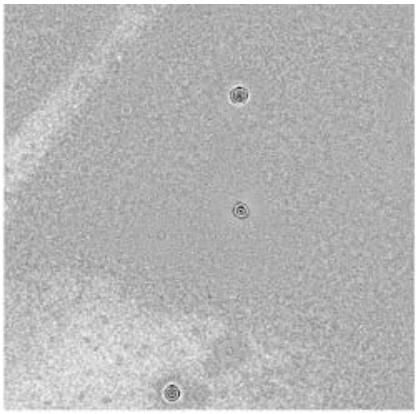

(b)
Fig. 2. Final products of the multiscale image processing involved when removing in a non-parametric way objects within a given scale range from an image. Images are $1.75^{\prime} \times 1.75^{\prime}$ in the $R$ band. a) Denoised image of the whole set of small scale structures in the original image as obtained by adding together the two images restored from the significant wavelet coefficients of the raw and residual images; b) difference between the original image and the image of the objects, showing that, except from a few annular artefacts due to local saturation, only noise and very large scale components are present in this second residual image.

larger than 512 pixels are therefore preserved in the first residual image, as well as a number of previously smaller hidden features.

The map of these remaining small scale structures has been obtained by means of a wavelet transform of this first residual with a computation implying only the first six, seven or eight scales of the dyadic $a=2^{j}$ scheme for checking purposes. A smaller set of scales than before is prefered so as to preserve in the final residual image most of the diffuse emission regions. For example, using the scale combination 10-6 (a multiscale examination with 10 and 6 scales at the first and second iteration, respectively), only the smallest diffuse sources appear on the object image, along with galaxies of various sizes, whereas large emission regions like the haloes of dominant galaxies or extended diffuse light appear on the final residual image. The scale combinations $10-7$ and 10-8 remove from the residual image the sources that are not very extended (i.e. with a typical diameter smaller than $2^{7}=128$ and $2^{8}=256$ pixels, respectively). Scale combinations $10-6,10-7$ and $10-8$ will roughly preserve objects larger than $40^{\prime \prime}$ (about 3 times the characteristic scale), $1.5^{\prime}$ and $3^{\prime}$ respectively.

The various steps of our analysis are shown for an example region in Figs. 1 and 2. This region corresponds to source 3 as described in the following.

\section{Results for the $\boldsymbol{R}$ band data}

\subsection{0-7 and 10-8 combination scales for the whole field of view: General overview}

We first describe in this section the results of the treatment for the whole field of view using two scale combinations: 10-7 and $10-8$. We analyzed separately the northern and southern mosaics. These mosaics overlap in the NGC 4874 and NGC 4889 area. We show the results with the 10-7 scale combination for the southern mosaic in Fig. 3 (reconstructed and 


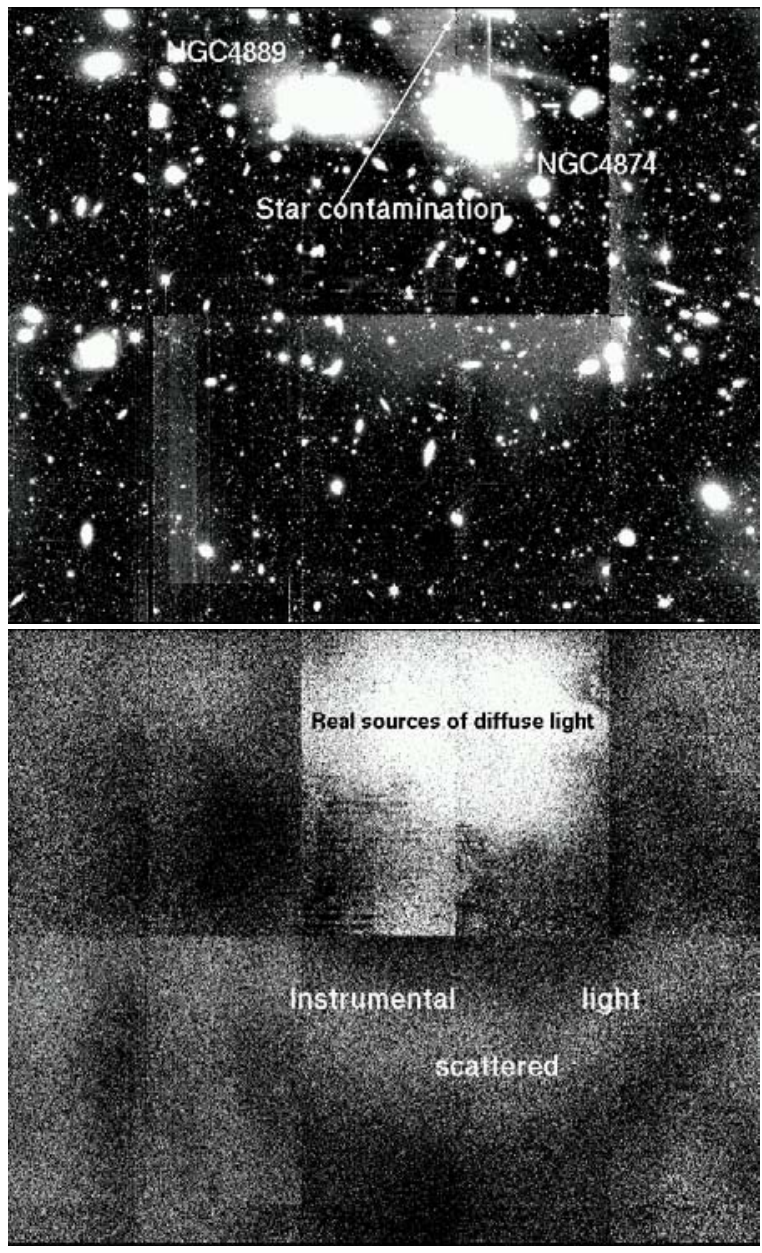

Fig. 3. Southern mosaic in the $R$ band. Upper figure: reconstructed image of objects computed with the scale combination 10-7. Lower figure: residual image computed with the scale combination $10-7$. We clearly see strong diffuse light emission towards the top of the lower image. The hemispheric structure corresponding to the light scattered by the instrument is visible in the bottom part of the lower image. North is up and East is left.

residual images) and for the northern mosaic in Fig. 4 (residual image).

We removed from the northern mosaic the areas around two bright stars located in the northwest part of the mosaic. Instrumental scattered light from these objects was so intense that it affected our treatment quite adversely. We only kept the area around the brightest star (just north of NGC 4874) because the main signal we detected was close to these coordinates.

In Figs. 3 and 4 we see a significant emission in the residual images around the dominant galaxies. This emission appears with both scales (10-7 and 10-8), and shows various features corresponding to different characteristic emission scales. This emission is detected in both northern and southern mosaic images, assuring us that the detection is not due to an instrumental effect such as scattered light, since the same regions on the sky are not located at the same place on the camera in the northern and southern fields.

We detected a large diffuse emission around the bright star in the northern mosaic (instrumental effect impossible to

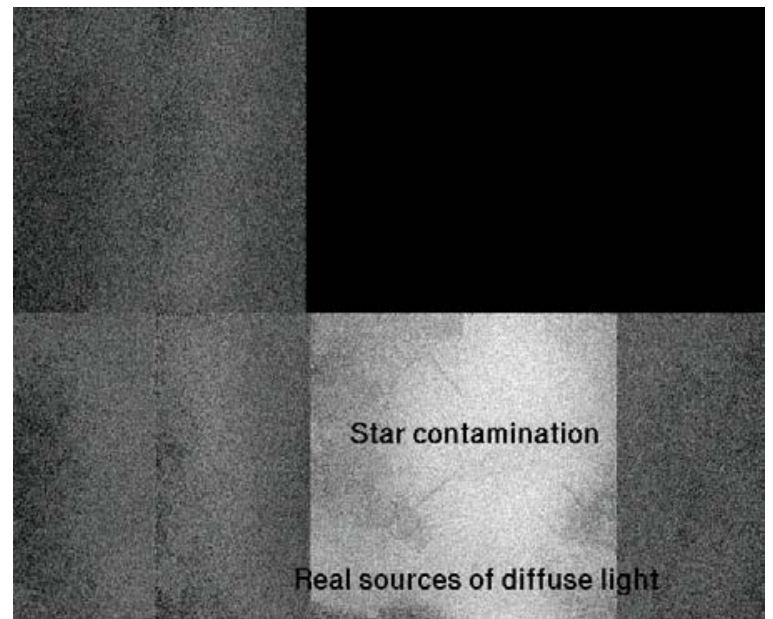

Fig. 4. Residual image of Northern mosaic computed with the scale combination $10-7$ in the $R$ band. In the bottom part of the image we clearly see diffuse emission in the region of galaxies NGC 4874 and 4889. Just above this diffuse emission, we can see the halo corresponding to the light of the bright star scattered by the instrument. The rectangle at the upper right was not considered because it contained two bright stars. North is up and East is left.

remove with our method without removing smaller scale diffuse light sources). We will therefore use preferentially the southern data to study the diffuse emission in the dominant galaxy area because these data are much less affected by bright stars. The diffuse light coming from the star located in the northern mosaic is still visible in the southern mosaic, but our treatment interprets this contribution as an object (it is visible in the object image) and removes it from the residual image. Contamination by this star in the southern mosaic is therefore negligible.

We detected in the southern field a nearly circular structure centered on the field center and mainly visible in the south part of the southern field. This is the light scattered by the instrument itself (see the CFHT database). This structure is annular with a central emission right in the middle of the field (it is indicated as "instrumental scattered light" in Fig. 3). It is not detected in the northern field because of the areas removed around the two bright stars and because of the large scale halo of the brightest star of the field.

\subsection{0-6, 10-7 and 10-8 combination scales for the dominant galaxy cluster region}

We study here a region of $14 \times 14 \operatorname{arcmin}^{2}$ around NGC 4874 and NGC 4889. In order to detect smaller scale structures in this region we also use a third scale combination: 10-6. The results are shown in Figs. 5-8.

These figures show the residual images with several characteristic positions: dominant galaxy positions, center of the bulk of the X-ray emission from the cluster (Neumann et al. 2003), center of the faint-galaxy density map (Biviano et al. 1996), dynamical center of the galaxy group associated to NGC 4874 (Gurzadyan \& Mazure 2001) and a secondary X-ray peak (Finoguenov 2004, private communication). Figure 8 shows two 3D maps with two view angles. 


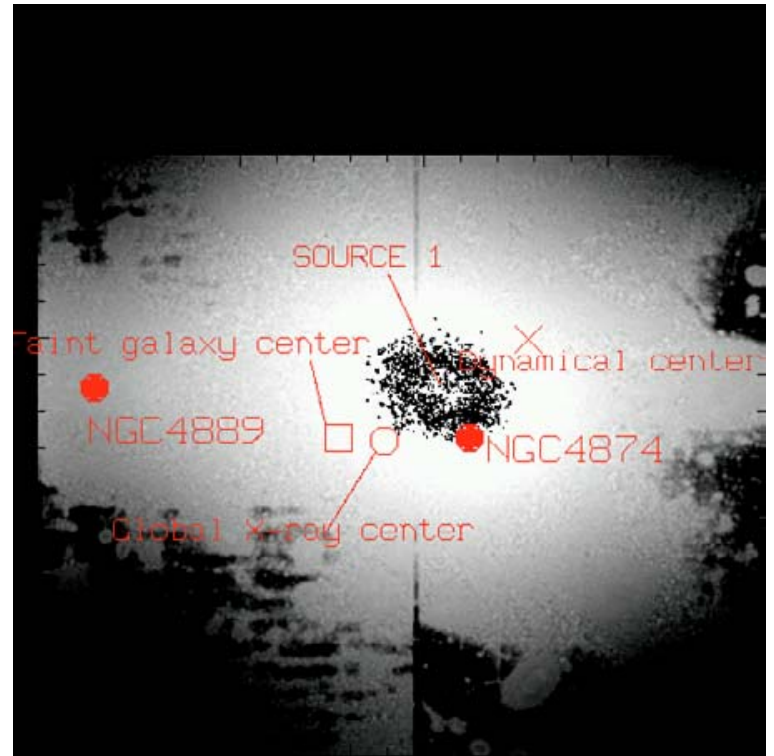

Fig. 5. $R$ band residual image of the area containing the two dominant galaxies computed with the 10-8 scale combination. Additional locations are shown: dynamical center of the NGC 4874 group from Gurzadyan \& Mazure (2001)(cross), faint galaxy center from Biviano et al. (1996)(square) and global X-ray center (open circle). Blackshaded areas show the diffuse light emission maximum (source 1). The two filled circles are the positions of the two cluster dominant galaxies. The image size is $14 \times 14 \mathrm{arcmin}^{2}$, as in the three following figures.

The 10-8 scale combination shows a single roughly circular large scale emission located north of NGC 4874, hereafter source 1 (see Fig. 5 and Table 1). This source does not seem to be associated with any object but NGC 4874. There is however a significant shift between NGC 4874 and the center of this emission. We note that we do not detect any such emission close to NGC 4889. It is therefore very unlikely that this source is a numerical artefact due to the presence of a bright galaxy (NGC 4874) nearby. Figure 9 shows maps of source 1 for the 10-6, 10-7 and 10-8 combination scales.

Using smaller scales (10-7), we find evidence for two other emissions in addition to the previous source (see Fig. 6 and Table 1). One of them (hereafter source 2) is located between NGC 4874 and NGC 4889. This emission is centered $\sim 2$ arcmin from the center of the faint galaxy density map (Biviano et al. 1996). Figure 10 shows maps of source 2 for the $10-6,10-7$ and 10-8 combination scales.

The other emission (hereafter source 3 ) coincides exactly with the center of the secondary X-ray emission (Finoguenov, private communication) and north west of the dynamical center of the NGC 4874 group. Looking at the image of the object, we see that this source is associated with the "plume" already reported by Gregg \& West (1998). Figure 11 shows maps of source 3 for the 10-6, 10-7 and 10-8 combination scales.

Using scales 10-6, we detect several sources (Figs. 7 and 8). One is associated with source 2. This means that source 2 has multiscale components. This component of source 2 nearly coincides with the center of the cluster bulk X-ray emission (Neumann et al. 2003). We also detect a source

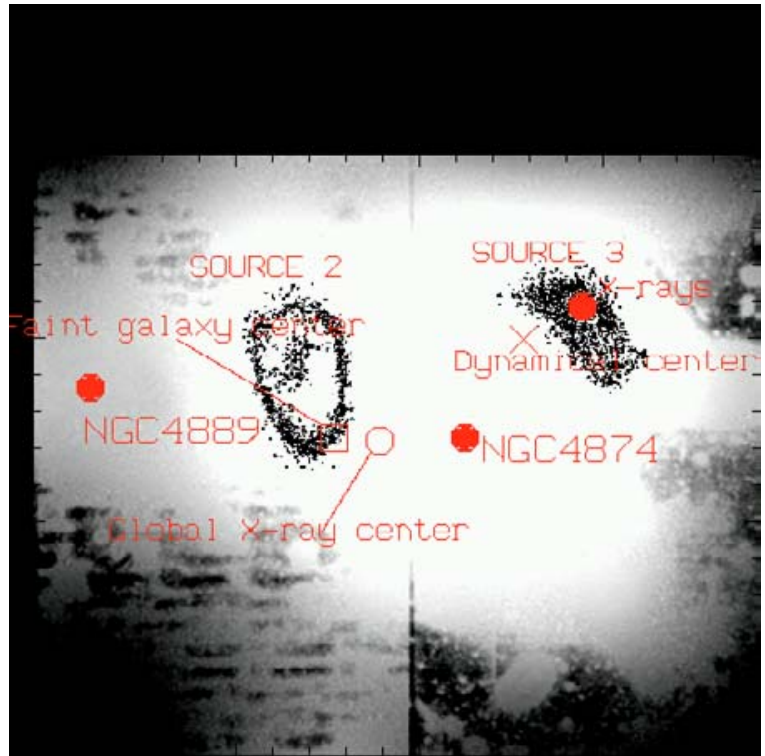

Fig. 6. $R$ band residual image of the area containing the two dominant galaxies computed with the 10-7 scale combination. Additional locations are shown as in Fig. 5, together with the secondary X-ray peak from Finoguenov (2004, private communication)(right filled circle). Black-shaded areas show the diffuse light emission maxima, with sources 2 (left) and 3 (right).

north of NGC 4874 (hereafter source 4). This source is possibly associated with a single galaxy even if no clear sign of galaxy disruption appears in the $R$ band image. This source is only visible with the 10-6 combination scale (see Fig. 12).

Finally, several sources are detected north and south of source 3 , implying that source 3 has multiscale components. The southern part of the small scale component of source 3 is located very close to the dynamical center of the NGC 4874 group.

\subsection{Statistical significance of the detections of sources 1, 2, 3 and 4}

In order to estimate the statistical significance of the detections of the four sources, we computed the signal-to-noise maps in the dominant galaxy cluster region of the $R$ band residual images (see Fig. 13). The contours overplotted on these images show respectively the 4 and $5 \sigma$ levels for the 10-6 scale combination, the 3.5 and $4 \sigma$ levels for the 10-7 scale combination and the 3 and $3.5 \sigma$ levels for the 10-8 scale combination. We clearly see that sources 2,3 and 4 are detected above the $4 \sigma$ level with the $10-6$ scale combination. Using the 10-7 scale combination, sources 2 and 3 are detected above the $3.5 \sigma$ level. Source 1 is also detected above the $3 \sigma$ level using the $10-8$ scale combination.

Therefore these four sources appear to be significantly detected.

\subsection{Comparison with the literature}

To assess the quality of our treatment, we compared our results with those of Gregg \& West (1998). They have detected three 

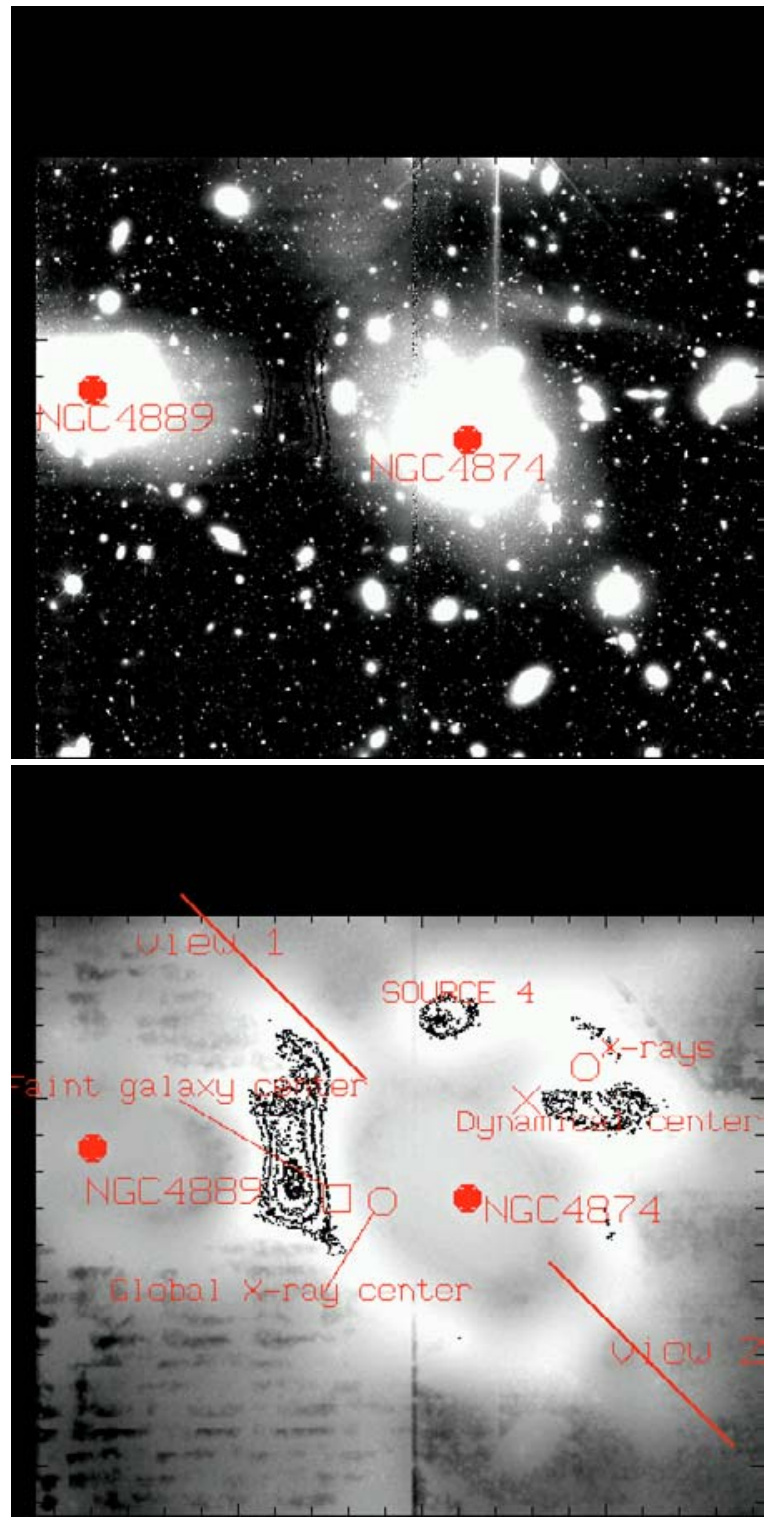

Fig. 7. $R$ band images. Upper figure: object image of the area containing the two dominant galaxies computed with the 10-6 scale combination. Lower figure: residual image of the area containing the two dominant galaxies computed with the 10-6 scale combination. Additional locations are shown as in Figs. 5 and 6. The two view directions of Fig. 8 are indicated. Black shaded areas show the diffuse light emission maxima, with source 4 north of NGC 4874 . The secondary X-ray peak from Finoguenov (2004, private communication) is shown as the open circle on the right.

small scale sources of diffuse light and one large scale source. Searching in our data in the same positions, we redetected what we call source 3 (source 1 of Gregg \& West: the largest scale source they detected). This source is brighter in our data because since we detect this source at a much larger scale than Gregg \& West (1998) we integrate more flux. The three other sources of Gregg \& West (1998) are not present in our residual images. However, we were not expecting to detect them in the residual images since we only search for large scale features. These three additional sources have very small scales and even the 10-6 scale combination removes them from the residual

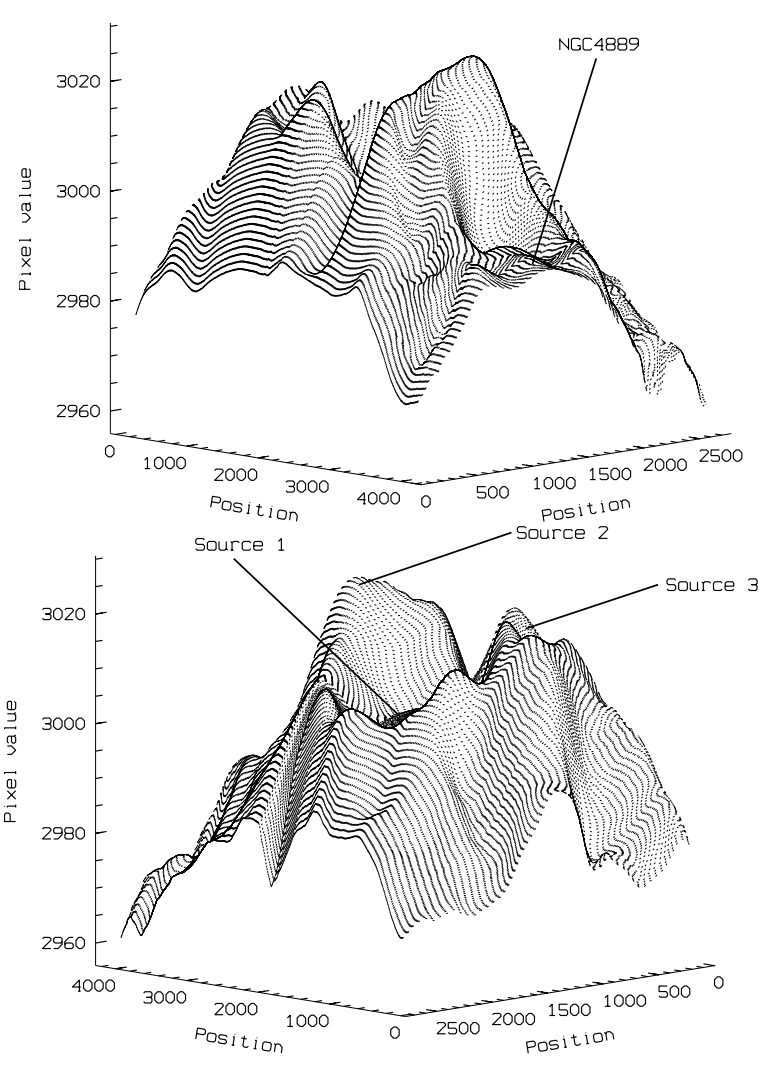

Fig. 8. $R$ band images. Upper figure: view 1 of residual image (10-6 combination scale) in Fig. 7. Lower figure: view 2 of residual image (10-6 combination scale) in Fig. 7. Angles of view are shown in Fig. 7. Additional locations of our sources and of NGC 4889 are shown.

images. This is confirmed by the fact that these three sources are indeed visible in the final object images.

For the same reason, we do not detect the same diffuse light concentrations as Bernstein et al. (1995). Their field was directly south of NGC 4874 and all their detected diffuse light was part of the $\mathrm{cD}$ halo. At this scale, these sources have been removed from our residual images.

\section{Results in the $B$ and $V$ bands}

\section{1. $B$ and $V$ bands}

Results of the analyses of the region of the dominant galaxies are shown in Figs. 14-16 for the $B, V$ and $R$ bands, and in Table 1. These figures show that source 1 is detected in $V$ and $R$ but is not visible in $B$, while sources 2 and 3 are detected in $B$, $V$ and $R$, and source 4 is only visible in $R$. Using combination scale 10-7 with $V$ band data leads to results similar to those obtained in the $R$ band.

\subsection{Detection limits and colors of the various sources of diffuse emission}

We estimated the detection limits in the $B, V$ and $R$ bands assuming that we were not able to detect large scale concentrations of diffuse light exceeding the background level by less than $1 \%$. At this level, the surface brightness detection 
(a)

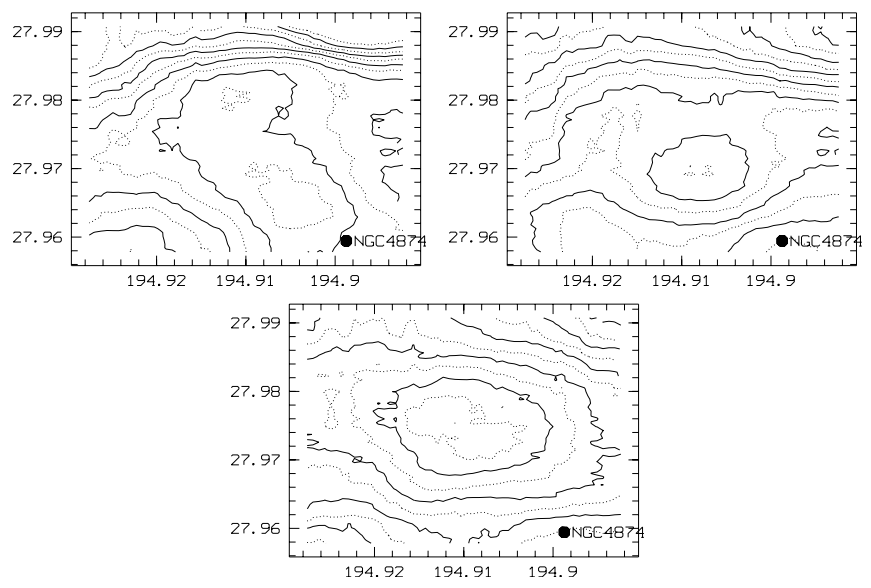

(c)

Fig. 9. Source 1 in the $R$ band for the 10-6 (a), 10-7 (b) and 10-8 (c) combination scales. (a)

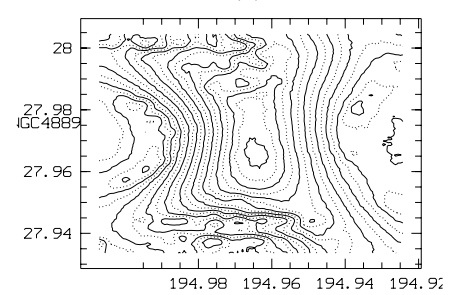

(b)

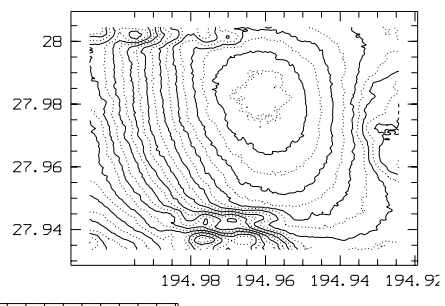

(c)

Fig. 10. Source 2 in the $R$ band for 10-6 (a), 10-7 (b) and 10-8 (c) combination scales.

limits are $25.4 \mathrm{mag} / \operatorname{arcsec}^{2}$ in $R, 25.8 \mathrm{mag} / \operatorname{arcsec}^{2}$ in $V$ and $27.2 \mathrm{mag} / \operatorname{arcsec}^{2}$ in $B$.

In order to measure colors for all four sources, we used the detections in the $R$ band and extracted the fluxes inside the same regions in the $V$ and $B$ bands. When the sources are not detected, colors are therefore only limits.

Considering the values in Table 1 , source 4 (with mean surface brightnesses of $R=25.1 \mathrm{mag} / \operatorname{arcsec}^{2}, V=$ $25.9 \mathrm{mag} / \operatorname{arcsec}^{2}$ and $B=26.4 \mathrm{mag} / \operatorname{arcsec}^{2}$ ) is detectable in $R$ and in $B$, but not in $V$. This explains why it is not visible in $V$. Since source 4 is not visible in $B$ either, it must be because this source is fainter in $B$ than in $R$ (that is, it is intrinsically red). Sources 1, 2 and 3 are detectable in the $B, V$ and $R$ bands (but source 1 is not detected in $B$ ).

We can also note that, even if sources 1,2 and 3 are detected in several bands, the locations of the emission peaks are shifted from one band to another, sometimes by a few arcminutes (see Figs. 9-16). We also applied the same computation to source 4 even if no significant concentration of diffuse light is present in

(a)

(b)
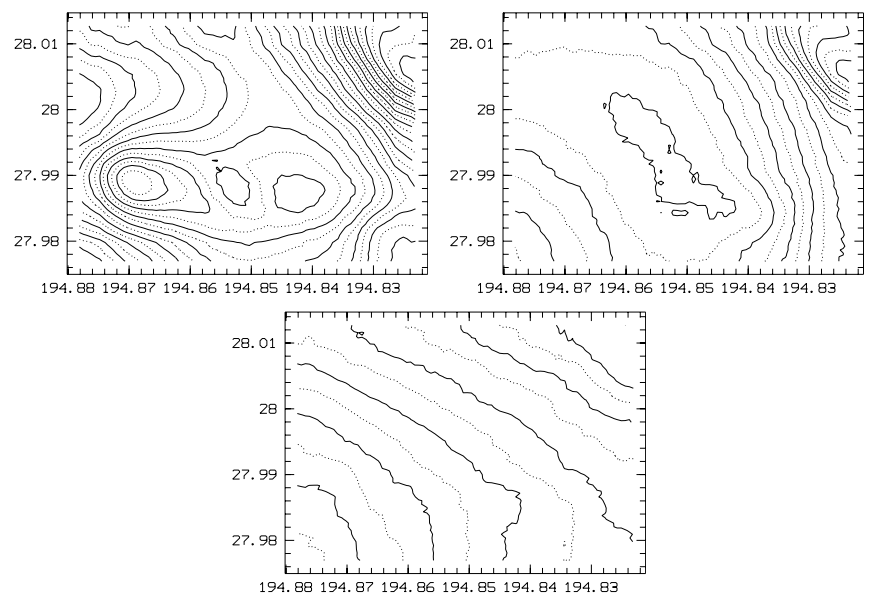

(c)

Fig. 11. Source 3 in the $R$ band for the 10-6 (a), 10-7 (b) and 10-8 (c) combination scales.

(a)

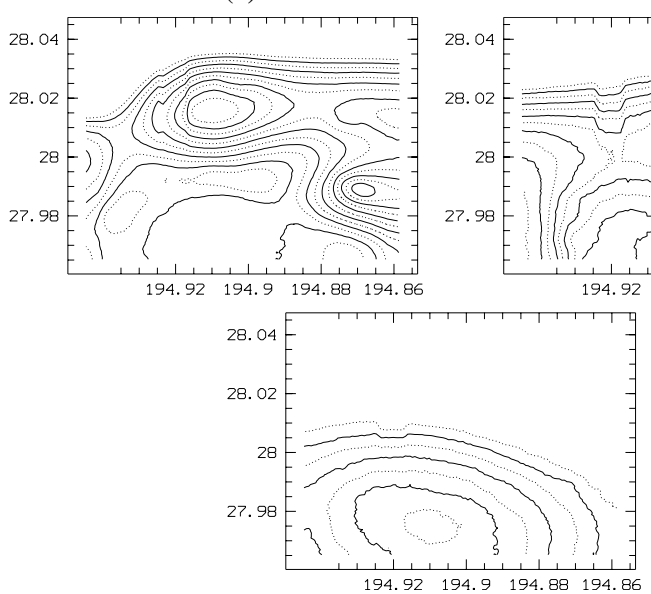

(b)

\section{(c)}

Fig. 12. Source 4 in the $R$ band for the 10-6 (a), 10-7 (b) and 10-8 (c) combination scales.

the $B$ and $V$ band data. Color computations are summarized in Table 1.

Sources 3 and 4 exhibit colors close to those of elliptical/S0 galaxies. Source 4 could be even redder, as the colors stated in Table 1 are only lower values. Therefore, these sources probably have moderate star forming activity. We note that we have similar results as Gregg \& West (1998) for source 3 given our estimated error bars.

Despite the large error bars, values in Table 1 suggest that source 2 has smaller $B-R$, more typical of a spiral-like than of an elliptical galaxy. Source 1 could possibly also have a spiral like color, but as we only have lower values for $B-R$ in Table 1, we cannot reach a definite conclusion for this source. This suggests non-negligible star forming activity at least inside source 2 . Finally, source 2 appears bimodal or elongated in the $B$ band (detected with the 10-7 combination scale) but not in the $V$ and $R$ bands, in agreement with the presence of extended stellar formation activity due to several discrete galaxy disruptions. 
Table 1. Characteristics of sources 1, 2, 3 and 4 detected with the 10-7 combination scales. $\alpha$ and $\delta$ are the J2000 central coordinates of the sources. Area is the (square) area used to compute the magnitudes and is given in arcmin ${ }^{2}$. The Gregg \& West (1998, GW98) values are also reported for source 3 .

\begin{tabular}{llllllllll}
\hline \hline Source & $\alpha$ & $\delta$ & Area & $V$ & $B-V$ & $B-V(\mathrm{GW} 98)$ & $V-R$ & $V-R(\mathrm{GW} 98)$ & $B-R$ \\
\hline 1 & 194.909 & 27.973 & 4.88 & $14.91 \pm 0.31$ & $\geq 0.67$ & & 0.42 & $\geq 1.09$ \\
2 & 194.964 & 27.966 & 2.82 & $15.36 \pm 0.34$ & 0.59 & & 0.48 & 1.07 \\
3 & 194.908 & 27.970 & 5.30 & $14.59 \pm 0.31$ & 0.93 & 0.93 & 0.36 & 0.57 & 1.29 \\
4 & 194.910 & 28.015 & 2.78 & $15.95 \pm 0.35$ & - & & $\geq 0.88$ & $\geq 1.30$ \\
\hline
\end{tabular}

(a)

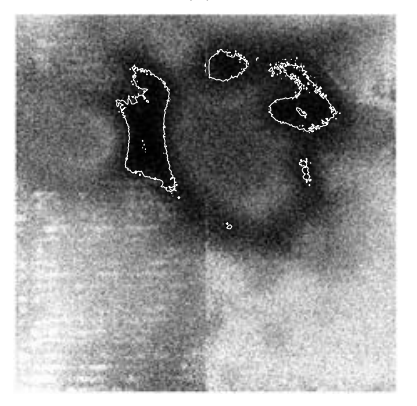

(b)

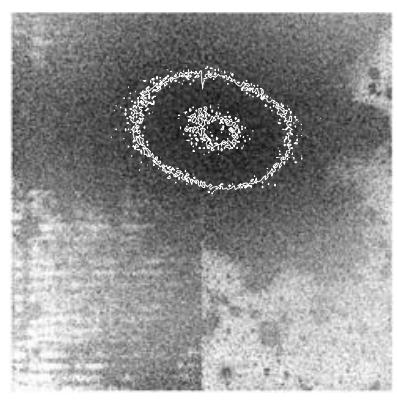

(a)
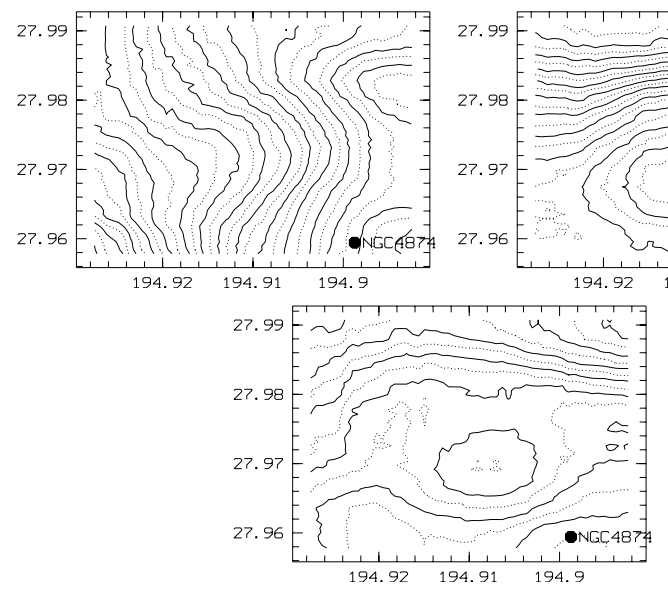

(c)

Fig. 14. Source 1 for the $10-7$ combination scale in the $B$ (a), $V$ (b) and $R$ (c) bands. (a)

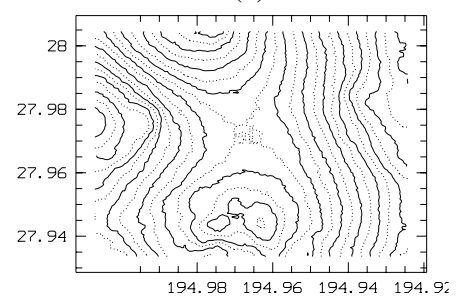

(b)

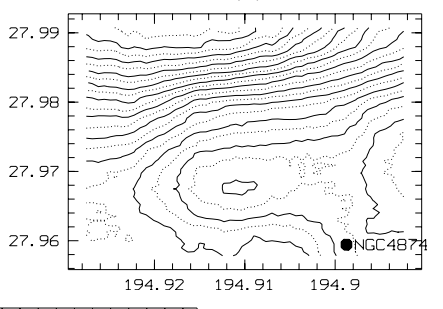

(c)

Fig. 13. $S / N$ maps for the 10-6 (upper left), 10-7 (upper right) and 10-8 (low center) scale combinations. Overlaid contours are for the 4-5 $\sigma$ levels (upper left), 3.5-4 $\sigma$ levels (upper right) and 3-3.5 $\sigma$ levels (low center).

\section{Towards a general scenario for the Coma cluster evolution?}

We assume in the following that diffuse light is coming from disrupted or harassed galaxies, a hypothesis which is supported by several studies (e.g. Gregg \& West 1998; Secker et al. 1998; Conselice \& Gallagher 1999; Adami et al. 2000).

\subsection{Dynamics of the cluster}

As discussed below, our results can help us to understand better the dynamics of the Coma cluster.

First, we do not detect any source of diffuse light around NGC 4889 while we have significant multi-scale sources around NGC 4874. This might have been the case if NGC 4889 had been present in the Coma cluster for a longer time than NGC 4874. NGC 4889 would then have had enough time to reaccrete the diffuse material produced for example by the disruption of a small galaxy or galaxy harassment. This is also supported by the fact that NGC 4874 has a central velocity dispersion higher than NGC $4889\left(398 \mathrm{~km} \mathrm{~s}^{-1}\right.$ against $275 \mathrm{~km} \mathrm{~s}^{-1}$,

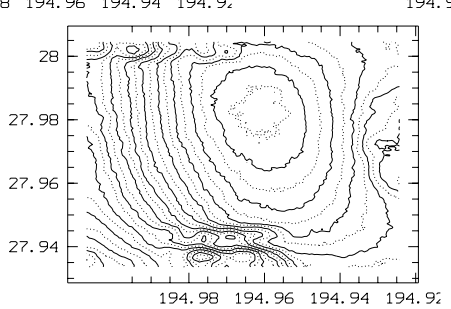

(b)

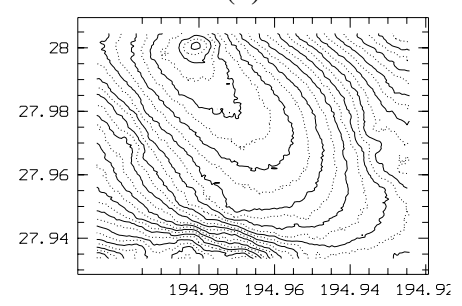

(c)

Fig. 15. Source 2 for the 10-7 combination scale in the $B$ (a), $V$ (b) and $R$ (c) bands.

Moore et al. 2002) and is also brighter. This tends to favor the notion that NGC 4889 has been in a dense environnement (the Coma cluster) for a longer time than NGC 4874.

Second, source 1 seems to be associated with NGC 4874 but is located north of the galaxy. Let us assume that NGC 4874 and its surrounding group are moving north through the Coma cluster (e.g. Donnelly et al. 1999). We know that NGC 4874 will lose its kinetic energy much more efficiently than all other 
(a)

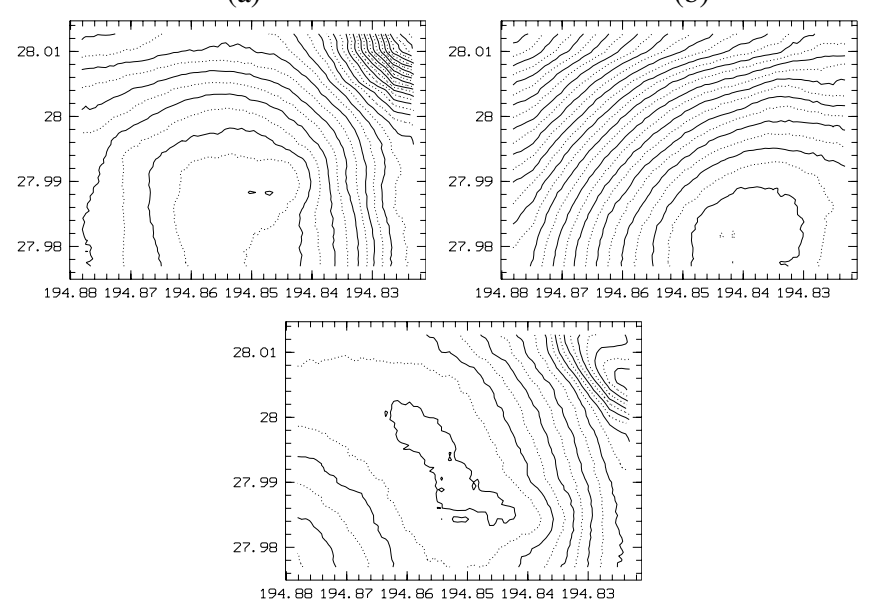

(c)

Fig. 16. Source 3 for the 10-7 combination scale in the $B$ (a), $V$ (b) and $R$ (c) bands.

galaxies in its group because of its large mass (e.g. Sarazin 1986). Hence, most of the galaxies of the NGC 4874 group will, therefore, be ahead of NGC 4874 towards the north. This is confirmed by the fact that the dynamical center of the NGC 4874 group is also located north of NGC 4874 (Gurzadyan \& Mazure 2001). We know that tidal disruptions will occur preferentially in low mass and late type galaxies. We also know that the NGC 4874 group has a quite late type galaxy content (Gurzadyan \& Mazure 2001). In this scenario, material dispersed by disruption or galaxy harassment from the NGC 4874 group will be located north of NGC 4874, as observed for example with sources 3 and 4 . Regarding source 3, one of the two galaxies involved in this disruption is indeed attached to the NGC 4874 group (Gurzadyan \& Mazure 2001). This motion towards the north is also confirmed by recent X-ray data. Donnelly et al. (1999) detected a hot region in the Coma cluster X-ray gas north of NGC 4874 using ASCA. This could be interpreted as a shock due to the motion of NGC 4874 through the Intra Cluster Medium. This detection was not confirmed in a first analysis of XMM data by Arnaud et al. (2001). However, Neumann et al. (2003) detected with the same XMM data a barely significant very elongated hot area located north of NGC 4874. This could therefore be the hot front associated with the motion of NGC 4874.

Source 2 has multiscale components and is located at equal distances from the two dominant Coma cluster galaxies. This is not surprising because it is the place where the tidal forces are the most intense in the cluster, and therefore where galaxy disruptions would occur. It is not clear, however, if this diffuse light source is associated with the Coma cluster potential itself or if it lies here only because of the influence of the two dominant galaxies. This area shows significant X-ray emission in excess of the global Coma cluster emission (e.g. Biviano et al. 1996; Neumann et al. 2003; Finoguenov et al. 2004, private communication) and this emission could be associated with the true Coma X-ray center. Similarly, Biviano et al. (1996) computed the position of the faint galaxy density map center and associated this center (close to the X-ray center computed by
Neumann et al. 2003) to the true Coma cluster center. However, the diffuse light source is not located exactly at the same place as shown in Figs. 6 and 7. The explanation probably lies in the multiscale content of source 2 . The small scale component (detected with the 10-6 scale combination) could be due to recent disruptions caused by the strong tidal forces in this region while the largest component (detected with the 10-7 scale combination) could simply be the result of older tidal disruptions retained at this place because it is close to the bottom of the Coma cluster potential. This does not explain, however, why the large scale component of source 2 is located significantly north of the Coma cluster center (faint galaxy center and global $\mathrm{X}$-ray center). Additional studies including numerical simulations are needed in order to confirm or invalidate this scenario.

\subsection{The nature of the diffuse light}

Assuming that all this large scale diffuse light is coming from totally disrupted galaxies, we computed the magnitude of a single galaxy able to produce such an amount of diffuse light. For the 10-7 scale combination, we would need an $R \sim 13$ or $M_{R} \sim-22$ galaxy (or $\sim 3 \times 10^{11} M_{\odot}$ assuming the same $M / L$ ratio as for the Milky Way). This is a galaxy brighter than $M^{*}$ and is comparable to the calculation of Gregg \& West (1998), who estimated that an $M_{R}=-22$ galaxy would produce the whole detected amount of small scale diffuse light in the Coma cluster. This is not, however, our favorite scenario as small galaxies are easier to disrupt than massive ones.

We also note that all this diffuse light represents only a negligible fraction of the total Coma luminosity (e.g. Girardi et al. 2002). However, if we limit ourselves to the cluster core (where the diffuse light is concentrated), the fraction becomes more important. In the very center, it represents up to $20 \%$ of the cluster galaxy luminosity. Assuming that the central $M / L$ ratio of Coma varies from 10 to 100 (e.g. Bernstein et al. 1995), it lowers the $M / L$ values by $15 \%$.

All these estimates assume that there is no significant ongoing star formation in the material emitting the diffuse light. Gregg \& West (1998) computed a color for source 3 close to that of an elliptical galaxy. This means that this diffuse light source is probably not forming significant amounts of stars. As mentionned above (See Table 1 and Sect. 5.2), to estimate the star formation rate in the various sources in which we detected diffuse light emission, we used our data to compute colors. To summarize the results: on the one hand, we confirm the results of Gregg \& West (1998, based on HST observations) for source 3 ; source 4 is also probably a quiet star forming region given its colors (probably even redder than the lower values given in Table 1). On the other hand, source 2 (and possibly source 1 ) has low $B-R$ colors (blue), typical of a spiral galaxy (e.g. Karick et al. 2003). We note that this last result is only marginally significant given the large uncertainties in Table 1 . The extent of source 2 in the $B$ band is larger (and bimodal) than in other bands. This suggests star forming activity in the outskirts of this source.

To explain these results we propose the following scenario: spiral galaxies are disrupted or harassed very efficiently by the 
combined tidal forces of NGC 4874 and NGC 4889 at the location of source 2 (e.g. Conselice et al. 2003). This leads to an abundant infall of still star-forming material in regions close to the bottom of the Coma cluster gravitational potential. This potential probably confines the matter at its present place. In agreement with this scenario, Stickel et al. (1998), using ISO data, detected a significant amount of dust located exactly where we detect source 2 while source 1 was less evident in their data. This is in good agreement with the less clear nature of source 1 given its marginally blue color (see Table 1 ). They concluded that a total amount of dust between $6.2 \times 10^{7}$ and $1.6 \times 10^{9} M_{\odot}$ would explain their observations. Assuming a dust to gas ratio between $1.3 \times 10^{-5}$ and $3.2 \times 10^{-4}$ (Stickel et al. 1998), we would predict a gas mass of between $1.9 \times 10^{11}$ and $1.2 \times 10^{14} M_{\odot}$. This is close to our estimate of the equivalent of one bright galaxy needed to generate the detected diffuse light. Moreover, Stickel et al. (1998) concluded that since dust is destroyed continuously by the hot ICM, the dust detected at the location of source 2 would be due to quite recent galaxy stripping. This is in good agreement with the hypothesis that source 2 has a recent component (appearing only in the 10-6 combination scale) originating from recent tidal disruptions or galaxy stripping due to the intense tidal forces between the two dominant galaxies. This would occur easily even for late type galaxies, generating diffuse light with a low $B-R$ color (blue), as observed in our data.

\section{Conclusions}

By applying a multi scale wavelet analysis and reconstruction technique to large images of the Coma cluster in various bands, we have shown the existence of several large scale sources of diffuse emission. The quality of the results obtained shows the power of this method for detecting faint diffuse emission in a rather crowded field.

No source of diffuse emission is detected around NGC 4889, possibly suggesting that this galaxy is older and has had time to accrete any diffuse material that may have been available in its surroundings.

On the other hand, we detected four diffuse sources around NGC 4874. Sources 3 and 4 have colors typical of early type galaxies, while source 2 probably shows signs of star formation activity, mainly in the outskirts of the concentration.

We suggest that a possible explanation for the origin of diffuse emission is that between the two main galaxies NGC 4874 and NGC 4889, spiral galaxies have recently been disrupted, and matter still forming stars has accumulated at the bottom of the cluster potential well. Other sources with colors typical of elliptical galaxies have probably been generated by older disruptions or disruptions of early type galaxies.

The characteristics of the sources of diffuse emission agree with a scenario in which the group around NGC 4874 is moving northwards, as suggested by recent $\mathrm{X}$-ray data.

Acknowledgements. The authors thank the referee for useful and constructive comments, J. Katgert for english corrections, M. Trayna for efficient numerical support and Olivier Ilbert for useful discussions.

\section{References}

Adami, C., Ulmer, M., Durret, F., et al. 2000, A\&A, 353, 930

Arnaboldi, M., Freeman, K. C., Mendez, R. H., et al. 1996, ApJ, 472, 145

Arnaboldi, M., Freeman, K. C., Okamura, S., et al. 2003, AJ, 125, 514

Arnaud, M., Aghanim, N., Gastaud, R., et al. 2001, A\&A, 365, L67

Bernstein, G. M., Nichol, R. C., Tyson, J. A., Ulmer, M. P., \& Wittman, D. 1995, AJ, 110, 1507

Bijaoui, A., \& Rué, F. 1995, Signal Process., 46, 345

Biviano, A., Durret, F., Gerbal, D., et al. 1996, A\&A, 311, 95

Calcáneo-Roldán, C., Moore, B., Bland-Hawthorn, J., Malin, D., \& Sadler, E. M. 2000, MNRAS, 314, 324

Conselice, C. J., \& Gallagher, J. S. 1999, AJ, 117, 75

Conselice, C. J., Gallagher, J. S., \& Wyse, R. F. G. 2003, AJ, 125, 66

Donnelly, R. H., Markevitch, M., Forman, W., et al. 1999, ApJ, 513, 690

Durrell, P. R., Ciardullo, R., Feldmeier, J. J., Jacoby, G. H., \& Sigurdsson, S. 2002, ApJ, 570, 119

Durret, F., Slezak, E., Lieu, R., Dos Santos, S., \& Bonamente, M. 2002, A\&A, 390, 397

Feldmeier, J. J., Ciardullo, R., \& Jacoby, G. H. 1998, ApJ, 503, 109

Feldmeier, J. J., Mihos, J. C., Morrison, H. L., Rodney, S. A., \& Harding, P. 2002, ApJ, 575, 779

Feldmeier, J. J., Mihos, J. C., Morrison, H. L., Harding, P., \& Kaib, N. 2003, Carnegie Observatories Astrophys. Ser., 3, Proc. Clusters of galaxies: probes of cosmological structure and galaxy evolution, ed. J. S. Mulchaey, A. Dressler, \& A. Oemler [arXiv: astro-ph/0303340]

Gal-Yam, A., Maoz, D., Guhathakurta, P., \& Filippenko, A. V. 2003, AJ, 125, 1087

Girardi, M., Manzato, P., Mezzetti, M., Giuricin, G., \& Limboz, F. 2002, ApJ, 569, 720

Gonzalez, A. H., Zabludoff, A. I., Zaritsky, D., \& Dalcanton, J. J. 2000, ApJ, 536, 561

Gregg, M. D., \& West, M. J. 1998, Nature, 396, 549

Gudehus, D. H. 1989, ApJ, 340, 661

Gurzadyan, V. G., \& Mazure, A. 2001, NewA, 6, 43

Karick, A. M., Drinkwater, M. J., \& Gregg, M. D. 2003, MNRAS, 344, 188

Mihos, J. C. 2003, Carnegie Observatories Astrophys. Ser., 3, Proc. Clusters of galaxies: probes of cosmological structure and galaxy evolution, ed. J. S. Mulchaey, A. Dressler, \& A. Oemler [arXiv: astro-ph/0305512]

Moore, A. W., Lucey, R. L., Kuntschner, H., \& Collless, M. 2002, MNRAS, 336, 382

Neumann, D. M., Lumb, D. H., Pratt, G. W., \& Briel, U. G. 2003, A\&A, 400, 811

Oemler, A., Jr. 1973, ApJ, 180, 11

Rué, F., \& Bijaoui, A. 1997, Exp. Astron., 7, 129

Sarazin, C. L. 1986, Rev. Mod. Phys., 58, 1

Secker, J., Harris, W. E., Cote, P., \& Oke, J. B. 1998, Proc. A new vision if an old cluster: untangling Coma Berenices, Marseille June 17-20, 1997, World Scientific, 115

Stickel, M., Lemke, D., Mattila, K., Haikala, L. K., \& Haas, M. 1998, A\&A, 329, 55

Theuns, T., \& Warren, S. J. 1997, MNRAS, 284, L11

Thuan, T. X., \& Kormendy, J. 1977, PASP, 89, 466

Trentham, N., \& Mobasher, B. 1998, MNRAS, 293, 53

Uson, J. M., Boughn, S. P., \& Kuhn, J. R. 1991, ApJ, 369, 46

Vílchez-Gómez, R., Pelló, R., \& Sanahuja, B. 1994, A\&A, 283, 37

Zwicky, F. 1951, PASP, 63, 61 